\title{
On a study of Sobolev type fractional functional evolution equations
}

\author{
Ismail T. Huseynov ${ }^{1}$, Arzu Ahmadova ${ }^{1}$, and Nazim Mahmudov ${ }^{1}$ \\ ${ }^{1}$ Eastern Mediterranean University
}

March 13, 2021

\begin{abstract}
Sobolev type fractional functional evolution equations have many applications in the modeling of many physical processes. Therefore, we investigate fractional-order time-delay evolution equation of Sobolev type with multi-orders in a Banach space and introduce an analytical representation of a mild solution via a new delayed Mittag-Leffler type function which is generated by linear bounded operators. Furthermore, we derive an exact analytical representation of solutions for multi-dimensional fractional functional dynamical systems with nonpermutable and permutable matrices. We also study stability analysis of the given time-delay system in Ulam-Hyers sense with the help of Laplace transform.
\end{abstract}

\section{Hosted file}

Sobolev-delay-last.pdf available at https://authorea.com/users/401265/articles/513414-on-astudy-of-sobolev-type-fractional-functional-evolution-equations 